\title{
Research on the Drug's Potential of Intrinsically Disordered Proteins
}

\author{
Peilun Jiang \\ College of Mobile Telecommunications Chongqing University of Posts and Telecom, Chongqing \\ 401520, China \\ billa@qq.com
}

\begin{abstract}
Intrinsically Disordered Proteins (IDPs) are a class of proteins that have no sure tertiary or secondary structure in the natural state. They are widely found in nature and involved in cell signal transduction, regulation, and protein interactions with a wide range of human diseases related. The research of IDPs provides new opportunities for drug design, and many cancer-related diseases are associated with IDPs lacking of ordered structures. However, the structural dynamic properties of IDPs present a significant obstacle to directly targets for drug design due to lacking of ordered structures in IDPs. This paper reviews the current research on the drug design progress of IDPs, lists examples on the drug design of IDPs, and some potential drug targets that can be used in IDPs. Finally, it analyzes the obstacles and difficulties in the drug design of IDPs.
\end{abstract}

Keywords: Intrinsically disordered proteins, drug design, drug targets, and molecular recognition.

\section{Introduction}

Protein is a kind of important biological macromolecules, plays a decisive role in the life process. Therefore, protein has been an important object of life science research for a long time. The research of protein science generally follows a paradigm of "sequence-structure-function" with the three-dimensional structure of protein as the kernel. The basic idea of this paradigm can be traced back to the lock-and-key model proposed by Fischer in 1894 [1]. Wu Xian [2] and Pauling [3] also respectively put forward the relevant theory in 1930s. The "sequence-structure-function" paradigm consider that the amino acid sequence of the protein determines the three-dimensional structure of the protein, and the three-dimensional structure of the protein determines the biological function of the protein.

However, in 1900s, scientists found that there is a class of proteins or protein's structural domain is in vitro physiological condition with little secondary structure. Such proteins are called Intrinsically Disordered Proteins (IDPs). IDPs lack a stable tertiary or secondary structure in natural physiological conditions. IDPs are not only widely found in nature, but also play an important role in the same function as ordered proteins, widely involved in signal transduction, DNA transcription, cell division, protein aggregation, and high selectivity of low selectivity Process [4-6].

Disordered proteins play an important role in processes, such as transcription and translation regulation, cell signal transduction, protein phosphorylation and small molecule storage. On the other hand, disorderly proteins seem to have some adverse effects on the biological system, It is often associated with a variety of diseases. In eukaryotes, the content of transcription factors is greater than $90 \%$ in the disordered protein fragment of length greater than 30 amino acids and up to $62.5 \%$ in E. coli. In addition, comprehensive use of structural characterization and biochemical methods for detailed research on specific proteins is an important direction of drug research [7].

\section{Drug Design of Intrinsically Disordered Proteins}

Disordered proteins flexible and complex disordered - orderly transition characteristics, enables it's to extensive interaction with other proteins and DNA. So, it can be combined with different receptors in many cases, and this one-to-many advantage allows the disordered protein to form a variety of structures after the combination. For example, in the C-terminal of transcription factor hypoxia-inducible factor-1 $\alpha$ (HIF $1 \alpha$ ) lacked of ordered structures, when the sequence binds to the zinc-binding TAZ1 $(\mathrm{CH} 1)$ domain of the cyclic AMP response element-binding protein (CREB) 
combined to form a helical structure; and after combine with asparagine hydroxylase that form a highly-stretched structure.

It is a good research direction that the design of small molecules hinders the formation of these unfavorable protein aggregates. Scientists can design small molecules to directly target the proper structure of such aggregated IDPs to prevent the occurrence of false folds, and to design non-toxic monomers or multimers for small molecules to stabilize such proteins, or design small molecules to directly accelerate the aggregation of such proteins, thereby minimizing the residence time of their toxic oligomers in the organism [8]. Now there are structural design based on these monomer's and oligomer's examples [9].

\section{Examples of Intrinsically Disordered Protein's Drug Design}

From the statistical data, $79 \%$ of the cancer-related proteins, $66 \%$ of the signal transduction proteins, $66 \%$ of the cardiovascular disease-related proteins contained more than 30 Residues of continuous disordered fragments, but this kind of protein in total protein's set that accounted for only 13\% [10-11]. And p53 associated with apoptosis and cancer, BRCA-1/2 proteins associated with breast cancer, c-Myc/Max [12-13] associated with various cancers, alpha- synuclein associated with neurodegenerative diseases, Tau and $\mathrm{A} \beta$ associated with Alzheimer's disease, and a series of prion proteins are either IDPs or have a greater degree of disorder.

AF9 is a Mixed Lineage Leukemia (MLL) fusion protein that causes carcinogenic transformation of hematopoietic cells. AF9 interacts with the most common fusion protein AF4 in acute leukemia. Bioinformatics analysis shows that fusion proteins usually exhibit a high degree of disordered structure. In the current example, both AF9 and AF4 are IDPs. Based on mapping studies, AF4-derived peptides have been developed to specifically interact with AF9 and demonstrate that AF4-AF9 interactions can be disrupted in vitro and in vivo [14]. Recently, AF9's nonpeptide inhibitors have also been successfully developed through high-throughput screening tests.

\section{Intrinsically Disordered Proteins as a Potential Drug Target}

Adapter molecule Crk (PDB ID 2EYY / 2EYZ) is also known as proto-oncogene c-Crk or p38. Which has several SH2 and SH3 structural domains and act as adapters for tyrosine kinases and small $\mathrm{G}$ proteins. It regulates survival, movement, proliferation and apoptosis during the process of transcription and cytoskeleton recombination of cells [15]. Crk overexpression has been identified as a cause of several human cancers, including breast cancer, ovarian cancer, lung cancer, brain cancer and gastric cancer. Therefore, the inhibition of Crk is an effective treatment for the treatment of these malignant tumors. For example, microRNAs have been used to attenuate Crk's translation process.

Proliferating cell nuclear antigen (PCNA) is an essential protein in DNA replication. A new type of protein p15PAF was identified as a PCNA-related factor by using PCNA as a bait in a yeast two-hybrid screening. p15PAF is located mainly in the nucleus, and p15PAF share sequences which bind to PCNA, with several other PCNA binding protein (including CDK inhibitor p21). And it will bind to $\mathrm{p} 21$-PCNA when $15 \mathrm{PAF}$ is overexpressed. Mutation of the sequence in p15PAF eliminates the binding activity of its PCNA. It is noteworthy that p15PAF was significantly increased expression in several types of tumors, especially in esophageal tumors. Like PCNA, p15PAF may have a wide range of implications in human cancers [16].

CRKI (SH2-SH3) and CRKII (SH2-SH3-SH3) are used to regulate transcription and cytoskeleton recombination for cell growth and movement by tyrosine kinase attachment to a small $\mathrm{G}$ protein. However, CRKII has a very low biological activity, phosphorylated CRKII is essentially non-bioactive, but CRKI exhibits significant biological activity. In conclusion, the molecular mechanisms are still elusive while underlying the different biological activities of CRK proteins. We determined the solution structure of CRKI, CRKII and phosphorylated CRKII by NMR, and identified the molecular mechanism that induced its activity. CRK plays an important role in regulating cell growth and motility in vivo, especially when it is a tyrosine kinase, which plays a role 
in transcriptional recombination during growth, movement, proliferation, differentiation and apoptosis. Thus, CRK is able to be an important effective therapeutic target possibly [17].

\section{Obstacles in the Drug Design of Intrinsically Disordered Proteins}

At present, the main obstacle is the lack of a complete set of strategic mechanisms in the drug's design of IDPs. The traditional rational drug designs are dependent on the background of the three-dimensional protein structure for ordered protein, and it is not feasible to apply the traditional design drug method directly to natural disorder proteins. The structural dynamic properties of IDPs present a significant obstacle to drug design that directly targets its disorder. However, IDPs are usually present in highly dynamic conformation, and it is difficult to determine the exact structure by experimental or theoretical means. For most of IDPs, it is impossible to use traditional methods for drug design. At present, there is still a lot of questions to be answered clearly about the paradigm relation of sequence-structure-function yet, and a lot of research work is needed. Thus, most of the drug design cases for IDPs were conducted through high-throughput experimental screening, not by rational design. Therefore, new strategies need to be developed to better rationalize drug design for IDPs.

\section{Summary}

In this research, we briefly discussed the discovery of intrinsically disordered proteins as well as the content in the biological body. The possibility was researched while as a drug design of IDPs. Moreover, we summarize examples of successful drug design examples, effective drug targets, and drug design with potentially targeted IDPs. And these research analyzes the difficulties and possibilities of the solution, when the IDPs is used in drug design, which is currently encountered by. Finally, it is only possible to develop a new strategy to deal with the high dynamic characteristics of IDPs in order to make a breakthrough in the virtual screening of IDPs drug design.

\section{References}

[1]. E. Fischer. Einfluss der Configuration auf die Wirkung der Enzyme. 1894. [J]. Berichte Der Deutschen Chemischen Gesellschaft, 2006, 27: 2985-2993.

[2]. H Wu. Studies on denaturation of proteins. XIII. A theory of denaturation. 1931. [J]. Advances in Protein Chemistry, 1995, 46(1): 6-26.

[3]. AE Mirsky, L Pauling. On the Structure of Native, Denatured, and Coagulated Proteins [J]. Proceedings of the National Academy of Sciences, 1936, 22: 439-447.

[4]. R Pushker, C Mooney, NE Davey, JM Jacque, DC Shields. Marked variability in the extent of protein disorder within and between viral families [J]. Plos One, 2013, 8: e60724.

[5]. DT Di, I Walsh, SC Tosatto. Analysis and consensus of currently available intrinsic protein disorder annotation sources in the MobiDB database [J]. BMC Bioinformatics, 2013, 14 Suppl 7(7): S3.

[6]. ME Oates, P Romero, T Ishida, M Ghalwash, MJ Mizianty, B Xue, Z Dosztanyi, VN Uversky, Z Obradovic, L Kurgan, AK Dunker, J Gough $\mathrm{D}^{2} \mathrm{P}^{2}$ database of disordered protein predictions [J]. Nucleic Acids Research, 2013, 41(Database issue): D508.

[7]. B Xue, AK Dunker, VN Uversky. Orderly order in protein intrinsic disorder distribution: disorder in 3500 proteomes from viruses and the three domains of life [J]. Journal of Biomolecular Structure \& Dynamics, 2012, 30:137-149.

[8]. VN Uversky. Intrinsically disordered proteins and novel strategies for drug discovery [J]. Expert Opinion on Drug Discovery, 2012, 475-488.

[9]. J Wang, Z Cao, L Zhao, S Li. Novel Strategies for Drug Discovery Based on Intrinsically Disordered Proteins (IDPs) [J]. International Journal of Molecular Sciences, 2011, 12(5): 3205-3219. 
[10]. Y Cheng, T LeGall, CJ Oldfield, JP Mueller, YYJ Van, P Romero, MS Cortese, VN Uversky, AK Dunker. Rational drug design via intrinsically disordered protein [J]. Trends in Biotechnology, 2006, 24(10): 435-42.

[11]. Metallo SJ. Intrinsically disordered proteins are potential drug targets [J]. Current Opinion in Chemical Biology, 14(4): 481-8.

[12]. N Issaeva, A Friedler, P Bozko, KG Wiman, AR Fersht, G Selivanova. Rescue of mutants of the tumour suppressor p53 in cancer cells by a designed peptide [J]. Proceedings of the National Academy of Sciences, 2013, 100(23): 13303.

[13]. LM Iakoucheva, CJ Brown, JD Lawson, Z Obradovic, AK Dunker. Intrinsic disorder in cell-signaling and cancer-associated proteins [J]. Journal of Molecular Biology, 323(3): 573-584.

[14]. RS Srinivasan, JB Nesbit, L Marrero, F Erfurth, VF LaRussa, CS Hemenway, The synthetic peptide PFWT disrupts AF4|[ndash]|AF9 protein complexes and induces apoptosis in $\mathrm{t}(4 ; 11)$ leukemia cells [J]. Leukemia. 2004, 18(8): 1364-72.

[15]. Y Kobashigawa, M Sakai, M Naito, M Yokochi, H Kumeta, Y Makino, K Ogura, S Tanaka, F Inagaki. Structural basis for the transforming activity of human cancer-related signaling adaptor protein CRK [J]. Tanpakushitsu Kakusan Koso Protein Nucleic Aci, 2007, 53(2): 503-510.

[16]. De Biasio A, Ibáñez de Opakua A, TN Cordeiro, M Villate, N Merino, N Sibille, M Lelli, T Diercks, P Bernadó, FJ Blanco. p15PAF is an intrinsically disordered protein with nonrandom structural preferences at sites of interaction with other proteins [J]. Biophysical Journal, 2014, 106(4): 865-874.

[17]. Y Kobashigawa, M Sakai, M Naito, M Yokochi, H Kumeta, Y Makino, K Ogura, S Tanaka, F Inagaki. Structural basis for the transforming activity of human cancer-related signaling adaptor protein CRK [J]. Tanpakushitsu Kakusan Koso Protein Nucleic Aci, 2007, 53(2):503-510. 